Commun. Korean Math. Soc. 25 (2010), No. 4, pp. 537-545

DOI 10.4134/CKMS.2010.25.4.537

\title{
HYPER MV-DEDUCTIVE SYSTEMS OF HYPER MV-ALGEBRAS
}

\author{
Young Bae Jun, Min Su Kang, and Hee Sik Kim
}

\begin{abstract}
The notions of (weak) hyper MV-deductive systems and (weak) implicative hyper MV-deductive systems are introduced, and several properties are investigated. Relations among hyper MV-deductive systems, weak hyper MV-deductive systems, implicative hyper MV-deductive systems and weak implicative hyper MV-deductive systems are discussed. A characterization of a hyper MV-deductive system is provided. A condition for a weak hyper MV-deductive system to be a weak implicative hyper MV-deductive system is given.
\end{abstract}

\section{Introduction}

In this paper, we introduce the notions of (weak) hyper MV-deductive systems and (weak) implicative hyper MV-deductive systems, and we investigates several properties. We discuss relations among hyper MV-deductive systems, weak hyper MV-deductive systems, implicative hyper MV-deductive systems and weak implicative hyper MV-deductive systems. We give a characterization of a hyper MV-deductive system. We give a condition for a weak hyper MV-deductive system to be a weak implicative hyper MV-deductive system.

\section{Preliminaries}

Definition 2.1 ([1]). A hyper $M V$-algebra is a non-empty set $M$ endowed with a hyper operation " $\oplus$ ", a unary operation " $*$ " and a constant " 0 " satisfying the following axioms:
(a1) $x \oplus(y \oplus z)=(x \oplus y) \oplus z$,
(a2) $x \oplus y=y \oplus x$
(a3) $\left(x^{*}\right)^{*}=x$,
(a4) $\left(x^{*} \oplus y\right)^{*} \oplus y=\left(y^{*} \oplus x\right)^{*} \oplus x$,
(a5) $0^{*} \in x \oplus 0^{*}$,
(a6) $0^{*} \in x \oplus x^{*}$,

Received December 15, 2009

2000 Mathematics Subject Classification. 06D35, 06F35, 03G25.

Key words and phrases. hyper MV-algebra, (weak) hyper MV-deductive system, (weak) implicative hyper MV-deductive system. 
(a7) $x \ll y, y \ll x \Rightarrow x=y$

for all $x, y, z \in M$, where $x \ll y$ is defined by $0^{*} \in x^{*} \oplus y$.

For every subsets $A$ and $B$ of $M$, we define

$$
\begin{gathered}
A \ll B \Leftrightarrow(\exists a \in A)(\exists b \in B)(a \ll b), \\
A \oplus B:=\bigcup_{a \in A, b \in B} a \oplus b .
\end{gathered}
$$

We also define $0^{*}:=1$ and $A^{*}:=\left\{a^{*} \mid a \in A\right\}$.

Proposition 2.2 ([1]). Every hyper $M V$-algebra $M$ satisfies the following statem-ents:

(b1) $(A \oplus B) \oplus C=A \oplus(B \oplus C)$,

(b2) $0 \ll x, \quad x \ll 1$,

(b3) $x \ll x$,

(b4) $x \ll y \Rightarrow y^{*} \ll x^{*}$

(b5) $A \ll B \Rightarrow B^{*} \ll A^{*}$,

(b6) $A \ll A$,

(b7) $A \subseteq B \Rightarrow A \ll B$,

(b8) $x \ll x \oplus y, \quad A \ll A \oplus B$,

(b9) $\left(A^{*}\right)^{*}=A$,

(b10) $0 \oplus 0=\{0\}$,

(b11) $x \in x \oplus 0$,

(b12) $y \in x \oplus 0 \Rightarrow y \ll x$,

(b13) $y \oplus 0=x \oplus 0 \Rightarrow x=y$

for all $x, y, z \in M$ and for all subsets $A, B$ and $C$ of $M$.

\section{Hyper MV-deductive systems}

In what follows, let $M$ denote a hyper MV-algebra unless otherwise specified.

Definition 3.1. A nonempty subset $D$ of $M$ is called a weak hyper $M V$ deductive system of $M$ if it satisfies:

(d1) $0 \in D$,

(d2) $(\forall x, y \in M)\left(\left(x^{*} \oplus y\right)^{*} \subseteq D, y \in D \Rightarrow x \in D\right)$.

Definition 3.2. A non-empty subset $D$ of $M$ is called a hyper $M V$-deductive system of $M$ if it satisfies (d1) and

(d3) $(\forall x, y \in M)\left(\left(x^{*} \oplus y\right)^{*} \ll D, y \in D \Rightarrow x \in D\right)$.

Example 3.3. Let $M:=\{0, a, b, 1\}$ be a set with a hyper operation " $\oplus$ " and a unary operation " $*$ " which are given in the following Cayley tables:

\begin{tabular}{|c|cccc|}
\hline$\oplus$ & 0 & $a$ & $b$ & 1 \\
\hline 0 & $\{0\}$ & $\{0, a\}$ & $\{0, a, b\}$ & $\{0, a, b, 1\}$ \\
$a$ & $\{0, a\}$ & $\{0, a\}$ & $\{0, a, b, 1\}$ & $\{0, a, b, 1\}$ \\
$b$ & $\{0, a, b\}$ & $\{0, a, b, 1\}$ & $\{0, a, b, 1\}$ & $\{0, a, b, 1\}$ \\
1 & $\{0, a, b, 1\}$ & $\{0, a, b, 1\}$ & $\{0, a, b, 1\}$ & $\{0, a, b, 1\}$ \\
\hline
\end{tabular}

\begin{tabular}{c|c}
$x$ & $x^{*}$ \\
\hline 0 & 1 \\
$a$ & $b$ \\
$b$ & $a$ \\
1 & 0
\end{tabular}


Then $\left(M, \oplus,^{*}, 0\right)$ is a hyper MV-algebra. It is easily checked that $D_{1}=\{0, a\}$, $D_{2}=\{0, b\}$ and $D_{3}=\{0, a, b\}$ are weak hyper MV-deductive systems of $M$.

Example 3.4. Consider the hyper MV-algebra $M$ which is described in Example 3.3. Then $D_{1}=\{0, a\}$ is a hyper MV-deductive system of $M$.

Example 3.5. Let $M:=\{0, a, b, 1\}$ be a set with a hyper operation " $\oplus$ " and a unary operation " $*$ " which are given in the following Cayley tables:

\begin{tabular}{|c|cccc|}
\hline$\oplus$ & 0 & $a$ & $b$ & 1 \\
\hline 0 & $\{0\}$ & $\{0, a\}$ & $\{0, b\}$ & $\{0, a, b, 1\}$ \\
$a$ & $\{0, a\}$ & $\{a\}$ & $\{0, a, b, 1\}$ & $\{0, a, b, 1\}$ \\
$b$ & $\{0, b\}$ & $\{0, a, b, 1\}$ & $\{0, a, b, 1\}$ & $\{0, a, b, 1\}$ \\
1 & $\{0, a, b, 1\}$ & $\{0, a, b, 1\}$ & $\{0, a, b, 1\}$ & $\{0, a, b, 1\}$ \\
\hline
\end{tabular}

\begin{tabular}{c|c}
$x$ & $x^{*}$ \\
\hline 0 & 1 \\
$a$ & $b$ \\
$b$ & $a$ \\
1 & 0
\end{tabular}

Then $\left(M, \oplus,{ }^{*}, 0\right)$ is a hyper MV-algebra. It is easily checked that $D_{1}=\{0, a\}$ is a hyper MV-deductive systems of $M$. But $D_{2}=\{0, b\}$ is not a hyper MVdeductive system of $M$, since $\left(a^{*} \oplus b\right)^{*}=\{0, a, b, 1\} \ll D_{2}, b \in D_{2}$ but $a \notin D_{2}$.

Example 3.6. Let $M=\{0, a, b, 1\}$ be a set with a hyper operation " $\oplus$ " and a unary operation " $*$ " which are given in the following Cayley tables:

\begin{tabular}{|c|cccc|}
\hline$\oplus$ & 0 & $a$ & $b$ & 1 \\
\hline 0 & $\{0\}$ & $\{0, a, b\}$ & $\{0, b\}$ & $\{0, a, b, 1\}$ \\
$a$ & $\{0, a, b\}$ & $\{0,1\}$ & $\{0, a, b, 1\}$ & $\{0, a, b, 1\}$ \\
$b$ & $\{0, b\}$ & $\{0, a, b, 1\}$ & $\{b\}$ & $\{0, a, b, 1\}$ \\
1 & $\{0, a, b, 1\}$ & $\{0, a, b, 1\}$ & $\{0, a, b, 1\}$ & $\{0, a, b, 1\}$ \\
\hline
\end{tabular}

\begin{tabular}{c|c}
$x$ & $x^{*}$ \\
\hline 0 & 1 \\
$a$ & $b$ \\
$b$ & $a$ \\
1 & 0
\end{tabular}

Then $\left(M, \oplus,^{*}, 0\right)$ is a hyper MV-algebra, but $D:=\{0, a, 1\}$ is not a weak hyper MV-deductive system of $M$, since $\left(b^{*} \oplus a\right)^{*}=(a \oplus a)^{*}=\{0,1\} \subseteq D$ and $b \notin D$. We can easily check that $D_{1}:=\{0, x\}, D_{2}:=\{0, b, x\}$ and $M$ are weak hyper MV-deductive systems of $M$ for all $x \in M$.

Proposition 3.7. Let $M$ be a hyper $M V$-algebra in which $0 \in x \oplus y$ for all $x, y \in M$. Let $D$ be a subset of $M$ such that $0 \in D$ and $1 \notin D$. Then $D$ is a weak hyper $M V$-deductive system of $M$.

Proof. For any $x, y \in M$, assume that $x \notin D$ and $y \in D$. Since $0 \in x^{*} \oplus y$, we have $1=0^{*} \in\left(x^{*} \oplus y\right)^{*}$, which shows that $\left(x^{*} \oplus y\right)^{*}$ is not contained in $D$. Therefore $D$ is a weak hyper MV-deductive system of $M$.

Theorem 3.8. Let $M$ be a hyper $M V$-algebra satisfying the following condition:

$$
(\forall x, y \in M)(x \in x \oplus y)
$$

Then every subset of $M$ containing 0 is a weak hyper $M V$-deductive system of $M$.

Proof. Let $D$ be a subset of $M$ containing 0 , and let $x, y \in M$ be such that $\left(x^{*} \oplus y\right)^{*} \subseteq D$ and $y \in D$. Assume that $x \notin D$. Then $x^{*} \in x^{*} \oplus y$ by (3.1), 
and so $x=\left(x^{*}\right)^{*} \in\left(x^{*} \oplus y\right)^{*}$ by (a3). Hence $\left(x^{*} \oplus y\right)^{*}$ is not contained in $D$, which is a contradiction. Therefore $x \in D$, proving that $D$ is a weak hyper MV-deductive system of $M$.

A hyper MV-algebra satisfying (3.1) can have a subset containing 0 which is not a hyper MV-deductive system of $M$. See the following example.

Example 3.9. Consider a hyper MV-algebra $M:=\{0, a, b, 1\}$ which is described in Example 3.5. It can be shown that $M$ satisfies (3.1). The set $D:=\{0, b\}$ is not a hyper MV-deductive system of $M$, since $\left(a^{*} \oplus b\right)^{*}=$ $\{0, a, b, 1\} \ll D$ and $a \notin D$.

Using (b7) and (d3), we have the following theorem.

Theorem 3.10. Every hyper $M V$-deductive system is a weak hyper $M V$-deductive system.

The following example shows that the converse of Theorem 3.10 is not valid.

Example 3.11. Consider a hyper MV-algebra $M$ which is described in Example 3.3. Then $D_{3}:=\{0, a, b\}$ is a weak hyper MV-deductive system of $M$ (see Example 3.3). We know that

$$
\left(1^{*} \oplus a\right)^{*}=(0 \oplus a)^{*}=\{0, a\}^{*}=\{b, 1\} \ll D
$$

and $a \in D$, but $1 \notin D$. This proves that $D_{3}$ is not a hyper MV-deductive system of $M$.

Proposition 3.12. Every hyper $M V$-deductive system $D$ of $M$ have the following condition:

$$
(\forall x, y \in M)(x \ll y, y \in D \Rightarrow x \in D) .
$$

Proof. Let $x, y \in M$ be such that $x \ll y$ and $y \in D$. Then $0 \in\left(x^{*} \oplus y\right)^{*}$, and so $\left(x^{*} \oplus y\right)^{*} \ll D$ by $(\mathrm{d} 1)$ and (b3). It follows from (d3) that $x \in D$.

Combining (b2) and Proposition 3.12, we have the following corollary.

Corollary 3.13. Let $D$ be a hyper $M V$-deductive system of $M$. If $D$ contains the element 1 , then $D=M$.

By Corollary 3.13, we know that any proper subset of $M$ containing 1 cannot be a hyper MV-deductive system of $M$.

Proposition 3.14. Let $D$ be a hyper $M V$-deductive system of $M$. For every subsets $A$ and $B$ of $M$, if $B \subseteq D$ and $\left(A^{*} \oplus B\right)^{*} \ll D$, then $A \ll D$.

Proof. We have $\left(A^{*} \oplus B\right)^{*}=\bigcup_{a \in A, b \in B}\left(a^{*} \oplus b\right)^{*} \ll D$, and so there exists $x \in\left(a^{*} \oplus b\right)^{*}$ for some $a \in A$ and $b \in B$, and there exists $y \in D$ such that $x \ll y$. It follows that $\left(a^{*} \oplus y\right)^{*} \ll D$. Since $b \in B \subseteq D$, by (d3) we obtain $a \in D$. Therefore $A \ll D$. 
Remark 3.15. (1) In Proposition 3.14, it is not necessary to mention that $A \subseteq$ $D$. To show this, consider a hyper MV-algebra $M=\{0, a, b, 1\}$ which is given in Example 3.5. Let $A:=\{0, a, b\}$ and $B:=\{0\}$. Note that $D:=\{0, a\}$ is a hyper MV-deductive system of $M$ and $\left(A^{*} \oplus B\right)^{*}=\{0, a, b, 1\} \ll D$, but $A$ is not contained in $D$.

(2) In Proposition 3.14, if we use $B \ll D$ instead of $B \subseteq D$, then the result does not hold. Consider a hyper MV-algebra $M=\{0, a, b, 1\}$ which is given in Example 3.5. It can be shown that $D:=\{0, a\}$ is a hyper MVdeductive system of $M$. If we take $A:=\{b\}$ and $B:=\{0, b\}$, then $B \ll D$ and $\left(A^{*} \oplus B\right)^{*}=\{0, a, b, 1\} \ll D$, but $A \ll D$ is not valid.

Proposition 3.16. Every weak hyper $M V$-deductive system $D$ of $M$ satisfies the following condition:

$$
(\forall A, B \subseteq M)\left(\left(A^{*} \oplus B\right)^{*} \subseteq D, B \subseteq D \Rightarrow A \subseteq D\right)
$$

Proof. For all $a \in A$ and $b \in B$, we have $\left(a^{*} \oplus b\right)^{*} \subseteq\left(A^{*} \oplus B\right)^{*} \subseteq D$ and $b \in B \subseteq D$. It follows from (d2) that $a \in D$.

Corollary 3.17. Every weak hyper $M V$-deductive system $D$ of $M$ satisfies the condition:

$$
(\forall A, B \subseteq M)\left(\left(A^{*} \oplus B\right)^{*} \subseteq D, B \subseteq D \Rightarrow A \ll D\right)
$$

Proof. Straightforward.

Remark 3.18. In Proposition 3.16, the condition $B \subseteq D$ can not be replaced by $B \ll D$. In fact, in Example 3.6, we see that if $A=\{b\}, B=\{a\}$ and $D=\{0,1\}$, then $D$ is a weak hyper MV-deductive system of $M$ and $B \ll D$. Also, $\left(A^{*} \oplus B\right)^{*}=\left(b^{*} \oplus a\right)^{*}=\{0,1\} \subseteq D$, but $A \nsubseteq D$.

We give a characterization of a hyper MV-deductive system.

Theorem 3.19. Let $D$ be a non-empty subset of $M$. Then $D$ is a hyper $M V$ deductive system of $M$ if and only if it satisfies (d1) and

$$
(\forall x, y \in M)\left(\left(x^{*} \oplus y\right)^{*} \cap D \neq \emptyset, y \in D \Rightarrow x \in D\right) .
$$

Proof. Assume that $D$ is a hyper MV-deductive system of $M$. Let $x, y \in M$ be such that $y \in D$ and $\left(x^{*} \oplus y\right)^{*} \cap D \neq \emptyset$. Then there exists $a \in\left(x^{*} \oplus y\right)^{*} \cap D$. Since $a \ll a$ by (b3), we get $\left(x^{*} \oplus y\right)^{*} \ll D$. It follows from (d3) that $x \in D$.

Conversely, suppose that $D$ satisfies (d1) and (e1). Let $x, y \in M$ be such that $y \in D$ and $\left(x^{*} \oplus y\right)^{*} \ll D$. Then there exist $a \in\left(x^{*} \oplus y\right)^{*}$ and $b \in D$ such that $a \ll b$. Hence $0^{*} \in a^{*} \oplus b$, and so $0 \in\left(a^{*} \oplus b\right)^{*}$. Since $0 \in D$, we have $0 \in\left(a^{*} \oplus b\right)^{*} \cap D$, i.e., $\left(a^{*} \oplus b\right)^{*} \cap D \neq \emptyset$. It follows from (e1) that $x \in D$. Therefore $D$ is a hyper MV-deductive system of $M$.

Proposition 3.20. Every hyper $M V$-deductive system $D$ of $M$ satisfies the following condition:

$$
(\forall A \subseteq M)(\forall b \in D)\left(\left(A^{*} \oplus b\right)^{*} \cap D \neq \emptyset \Rightarrow A \cap D \neq \emptyset\right)
$$


Proof. Let $A \subseteq M$ and $b \in D$ be such that $\left(A^{*} \oplus b\right)^{*} \cap D \neq \emptyset$. Then there exists $x \in D$ such that $x \in\left(a^{*} \oplus b\right)^{*}$ for some $a \in A$. Thus $\left(a^{*} \oplus b\right)^{*} \cap D \neq \emptyset$. Since $D$ is a hyper MV-deductive system, it follows from Theorem 3.19 that $a \in D$ so that $A \cap D \neq \emptyset$.

Theorem 3.21. For any hyper $M V$-deductive system $D$ of $M$, the following are equivalent:

(i) $(\forall x, y \in M)\left(\left(x^{*} \oplus y\right)^{*} \ll D\right)$.

(ii) $(\forall x, y \in M)\left(\left(x^{*} \oplus y\right)^{*} \cap D \neq \emptyset\right)$.

Proof. (i) $\Rightarrow$ (ii). Assume that (i) is valid. Then there exist $a \in\left(x^{*} \oplus y\right)^{*}$ and $b \in D$ such that $a \ll b$, that is, $0^{*} \in a^{*} \oplus b$. Thus $0 \in\left(a^{*} \oplus b\right)^{*} \subseteq\left(\left(x^{*} \oplus y\right) \oplus b\right)^{*}$, and so $\left(\left(x^{*} \oplus y\right) \oplus b\right)^{*} \cap D \neq \emptyset$. It follows from (b9) and Proposition 3.20 that $\left(x^{*} \oplus y\right)^{*} \cap D \neq \emptyset$.

(ii) $\Rightarrow$ (i). Straightforward.

\section{Implicative hyper MV-deductive systems}

Definition 4.1. A non-empty subset $D$ of $M$ is called a weak implicative hyper $M V$-deductive system of $M$ if it satisfies (d1) and

$$
(\forall x, y, z \in M)\left(\left(\left(x^{*} \oplus z\right) \oplus\left(y^{*} \oplus x\right)^{*}\right)^{*} \subseteq D, z \in D \Rightarrow x \in D\right) .
$$

Example 4.2. Let $M:=\{0, a, b, 1\}$ be a set with the hyper operation " $\oplus$ " and the unary operation " $*$ " which are given in the following Cayley tables:

\begin{tabular}{|c|cccc|}
\hline$\oplus$ & 0 & $a$ & $b$ & 1 \\
\hline 0 & $\{0\}$ & $\{0, a\}$ & $\{b\}$ & $\{b, 1\}$ \\
$a$ & $\{0, a\}$ & $\{0, a\}$ & $\{b, 1\}$ & $\{b, 1\}$ \\
$b$ & $\{b\}$ & $\{b, 1\}$ & $\{b, 1\}$ & $\{b, 1\}$ \\
1 & $\{b, 1\}$ & $\{b, 1\}$ & $\{b, 1\}$ & $\{b, 1\}$ \\
\hline
\end{tabular}

\begin{tabular}{c|c}
$x$ & $x^{*}$ \\
\hline 0 & 1 \\
$a$ & $b$ \\
$b$ & $a$ \\
1 & 0
\end{tabular}

Then $\left(M, \oplus,{ }^{*}, 0\right)$ is a hyper MV-algebra. For every $x \in M$, the set $\{0, x\}$ is a weak implicative hyper MV-deductive system of $M$. Also, $D_{1}:=\{0, b, 1\}$ is a weak implicative hyper MV-deductive system of $M$. We know that $D:=$ $\{0, a, b\}$ is not a weak implicative hyper MV-deductive system of $M$, since

$$
\left(\left(1^{*} \oplus b\right) \oplus\left(a^{*} \oplus 1\right)^{*}\right)^{*}=\{0, a\} \subseteq D
$$

and $b \in D$, but $1 \notin D$.

Definition 4.3. A non-empty subset $D$ of $M$ is called an implicative hyper $M V$-deductive system of $M$ if it satisfies (d1) and

$$
(\forall x, y, z \in M)\left(\left(\left(x^{*} \oplus z\right) \oplus\left(y^{*} \oplus x\right)^{*}\right)^{*} \ll D, z \in D \Rightarrow x \in D\right) .
$$

Example 4.4. In a hyper MV-algebra $M:=\{0, a, b, 1\}$ in Example 4.2, $\{0, a\}$ is an implicative hyper MV-deductive system of $M$. But $\{0, b\}$ is not an implicative hyper MV-deductive system of $M$, since

$$
\left(\left(1^{*} \oplus b\right) \oplus\left(a^{*} \oplus 1\right)^{*}\right)^{*}=\{0, a\} \ll\{0, b\} .
$$


Theorem 4.5. Every implicative hyper $M V$-deductive system is a weak implicative hyper $M V$-deductive system.

Proof. Let $D$ be an implicative hyper MV-deductive system of $M$ and let $x, y, z \in M$ be such that $z \in D$ and $\left(\left(x^{*} \oplus z\right) \oplus\left(y^{*} \oplus x\right)^{*}\right)^{*} \subseteq D$. Using (b7), we have $\left(\left(x^{*} \oplus z\right) \oplus\left(y^{*} \oplus x\right)^{*}\right)^{*} \ll D$. It follows from (d5) that $x \in D$. Therefore $D$ is a weak implicative hyper MV-deductive system of $M$.

The following example shows that the converse of Theorem 4.5 need not be true in general.

Example 4.6. Consider a hyper MV-algebra $M:=\{0, a, b, 1\}$ which is given in Example 3.3. Then it can be easily shown that every subset of $M$ containing 0 is a weak implicative hyper MV-deductive system of $M$, but $D:=\{0, a, 1\}$ is not an implicative hyper MV-deductive system of $M$, since

$$
\left(\left(b^{*} \oplus 0\right) \oplus\left(0^{*} \oplus b\right)^{*}\right)^{*}=M \ll\{0, a, 1\} .
$$

Theorem 4.7. Every implicative hyper $M V$-deductive system is a hyper $M V$ deductive system.

Proof. Let $D$ be an implicative hyper MV-deductive system of $M$ and let $x, y \in$ $M$ be such that $y \in D$ and $\left(x^{*} \oplus y\right)^{*} \ll D$. Then there exist $a \in\left(x^{*} \oplus y\right)^{*}$ and $z \in D$ such that $a \ll z$. Using (b2), (b9) and (b11), we obtain

$$
a^{*} \in a^{*} \oplus 0 \subseteq\left(\left(x^{*} \oplus y\right)^{*}\right)^{*} \oplus\left(0^{*} \oplus x\right)^{*}=\left(x^{*} \oplus y\right) \oplus\left(0^{*} \oplus x\right)^{*},
$$

and so $a \in\left(\left(x^{*} \oplus y\right) \oplus\left(0^{*} \oplus x\right)^{*}\right)^{*}$. Hence $\left(\left(x^{*} \oplus y\right) \oplus\left(0^{*} \oplus x\right)^{*}\right)^{*} \ll D$ which implies from (d5) that $x \in D$. Therefore $D$ is a hyper MV-deductive system of $M$.

The following example shows that the converse of Theorem 4.7 need not be true in general.

Example 4.8. Consider a hyper MV-algebra $M:=\{0, a, b, 1\}$ which is given in Example 3.3. Then $D:=\{0, a\}$ is a hyper MV-deductive system of $M$. Since

$$
\left(\left(1^{*} \oplus 0\right) \oplus\left(0^{*} \oplus 1\right)^{*}\right)^{*}=M \ll\{0, a\},
$$

$D=\{0, a\}$ is not an implicative hyper MV-deductive system of $M$.

The following example shows that a weak implicative hyper MV-deductive system may not be a weak hyper MV-deductive system.

Example 4.9. Consider a hyper MV-algebra $M:=\{0, a, b, 1\}$ which is described in Example 3.6. Then $D:=\{0, a, 1\}$ is a weak implicative hyper MVdeductive system of $M$, but it is not a weak hyper MV-deductive system of $M$, since $\left(b^{*} \oplus a\right)^{*}=\{0,1\} \subseteq D$.

We give a condition for a weak hyper MV-deductive system to be a weak implicative hyper MV-deductive system. 
Theorem 4.10. Let $D$ be a weak hyper $M V$-deductive system of $M$ satisfying the following condition:

$$
(\forall x, y \in M)\left(\left(x^{*} \oplus\left(y^{*} \oplus x\right)^{*}\right)^{*} \subseteq D \Rightarrow x \in D\right)
$$

Then $D$ is a weak implicative hyper $M V$-deductive system of $M$.

Proof. Let $x, y, z \in M$ be such that $z \in D$ and $\left(\left(x^{*} \oplus z\right) \oplus\left(y^{*} \oplus x\right)^{*}\right)^{*} \subseteq D$. Then

$$
\left(\left(\left(x^{*} \oplus\left(y^{*} \oplus x\right)^{*}\right)^{*}\right)^{*} \oplus z\right)^{*}=\left(\left(x^{*} \oplus\left(y^{*} \oplus x\right)^{*}\right) \oplus z\right)^{*} \subseteq D .
$$

Using Proposition 3.16, we obtain $\left(x^{*} \oplus\left(y^{*} \oplus x\right)^{*}\right)^{*} \subseteq D$ which implies from (4.1) that $x \in D$. This shows that $D$ is a weak implicative hyper MV-deductive system of $M$.

We provide a condition for a weak implicative hyper MV-deductive system to be a weak hyper MV-deductive system, as follows:

Theorem 4.11. Assume that $M$ satisfies the following condition:

$$
(\forall x \in M)\left(\left|\left(0^{*} \oplus x\right)^{*}\right|=1=\left|\left(x^{*} \oplus 0\right)^{*}\right|\right) .
$$

Then every weak implicative hyper $M V$-deductive system of $M$ is a weak hyper $M V$-deductive system of $M$.

Proof. If $M$ satisfies (4.2), then $\left(0^{*} \oplus x\right)^{*}=\{0\}$ and $\left(x^{*} \oplus 0\right)^{*}=\{x\}$ for all $x \in M$. Hence $\left(0^{*} \oplus A\right)^{*}=\{0\}$ and $\left(A^{*} \oplus 0\right)^{*}=A$ for every non-empty subset $A$ of $M$. Let $x, y \in M$ be such that $y \in D$ and $\left(x^{*} \oplus y\right)^{*} \subseteq D$. Then

$$
\left(\left(x^{*} \oplus y\right) \oplus\left(0^{*} \oplus x\right)^{*}\right)^{*}=\left(\left(x^{*} \oplus y\right) \oplus 0\right)^{*}=\left(x^{*} \oplus y\right)^{*} \subseteq D,
$$

which implies from (d4) that $x \in D$. Therefore $D$ is a weak hyper MV-deductive system of $M$.

Theorem 4.12. Every implicative hyper $M V$-deductive system $D$ of $M$ satisfies the following condition:

(e2) $\quad(\forall x, y, z \in M)\left(\left(\left(x^{*} \oplus z\right) \oplus\left(y^{*} \oplus x\right)^{*}\right)^{*} \cap D \neq \emptyset, z \in D \Rightarrow x \in D\right)$.

Proof. Assume that $D$ is an implicative hyper MV-deductive system of $M$. Let $x, y, z \in M$ be such that $z \in D$ and $\left(\left(x^{*} \oplus z\right) \oplus\left(y^{*} \oplus x\right)^{*}\right)^{*} \cap D \neq \emptyset$. Then there exists $a \in\left(\left(x^{*} \oplus z\right) \oplus\left(y^{*} \oplus x\right)^{*}\right)^{*} \cap D$. Since $a \ll a$ by (b3), we have $\left(\left(x^{*} \oplus z\right) \oplus\left(y^{*} \oplus x\right)^{*}\right)^{*} \ll D$. Using (d5), we obtain $x \in D$, which proves (e2).

Theorem 4.13. If a non-empty subset $D$ of $M$ satisfies (d1) and (e2), then $D$ is a hyper $M V$-deductive system of $M$.

Proof. Let $x, y \in M$ be such that $y \in D$ and $\left(x^{*} \oplus y\right)^{*} \cap D \neq \emptyset$. Then there exists $a \in M$ such that $a \in\left(x^{*} \oplus y\right)^{*}$ and $a \in D$. Using (b2), (b9) and (b11), we have $a^{*} \in a^{*} \oplus 0 \subseteq\left(x^{*} \oplus y\right) \oplus\left(0^{*} \oplus x\right)^{*}$ and so $a \in\left(\left(x^{*} \oplus y\right) \oplus\left(0^{*} \oplus x\right)^{*}\right)^{*}$. Hence $\left(\left(x^{*} \oplus y\right) \oplus\left(0^{*} \oplus x\right)^{*}\right)^{*} \cap D \neq \emptyset$, which implies from $(\mathrm{e} 2)$ that $x \in D$. Using Theorem 3.19 , we conclude that $D$ is a hyper MV-deductive system of $M$. 
Theorem 4.14. Let $D$ be a non-empty subset of $M$. If $D$ satisfies (d1) and (e2), then $D$ is an implicative hyper $M V$-deductive system of $M$.

Proof. Let $x, y, z \in M$ be such that $\left(\left(x^{*} \oplus z\right) \oplus\left(y^{*} \oplus x\right)^{*}\right)^{*} \ll D$ and $z \in D$. Then there exist $a, b \in M$ such that $a \in\left(\left(x^{*} \oplus z\right) \oplus\left(y^{*} \oplus x\right)^{*}\right)^{*}, \quad b \in D$ and $a \ll b$, i.e., $0 \in\left(a^{*} \oplus b\right)^{*}$. It follows that $\left(a^{*} \oplus b\right)^{*} \cap D \neq \emptyset$. By applying Theorems 3.19 and 4.13 we obtain that $a \in D$. Thus $\left(\left(x^{*} \oplus z\right) \oplus\left(y^{*} \oplus x\right)^{*}\right)^{*} \cap D \neq \emptyset$, which implies from (e2) that $x \in D$. Therefore $D$ is an implicative hyper MVdeductive system of $M$.

\section{References}

[1] Sh. Ghorbani, A. Hasankhani, and E. Eslami, Hyper MV-algerbas, Set-Valued Math. Appl. (to appear).

[2] Sh. Ghorbani, E. Eslami, and A. Hasankhani, Quotient hyper MV-algerbas, Sci. Math. Jpn. 66 (2007), 371-386.

[3] L. Torkzadeh and A. Ahadpanah, Hyper MV-ideals in hyper MV-algerbas, Math. Log. Quart. 56 (2010), 51-62.

Young Bae Jun

Department of Mathematics Education (and Rins)

Gyeongsang National University

Chinju 660-701, Korea

E-mail address: skywine@gmail.com

Min SU KANG

Department of Mathematics

HANYANG UNIVERSITY

Seoul 133-791, Korea

E-mail address: sinchangmyun@hanmail.net

Hee Sik Kim

Department of Mathematics

HANYANG UNIVERSITY

SeOUl 133-791, KoreA

E-mail address: heekim@hanyang.ac.kr 